\title{
The organization of spatial reference frames involved in the SNARC effect
}

\author{
Arnaud Viarouge ${ }^{1,2,3}$, Edward M. Hubbard ${ }^{2,3}$, and Stanislas Dehaene De,2,3 $^{1,4}$ \\ ${ }^{1}$ Cognitive Neuroimaging Unit, Université Paris-Sud, Orsay, France \\ ${ }^{2}$ INSERM, U992, Cognitive Neuroimaging Unit, CEA/SAC/DSV/DRM/Neurospin, Gif/Yvette, France \\ ${ }^{3} \mathrm{CEA}, \mathrm{DSV} / \mathrm{I} 2 \mathrm{BM}, \mathrm{Neurospin}$ Center, Gif/Yvette, France \\ ${ }^{4}$ Collège de France, Paris, France
}

\begin{abstract}
The SNARC effect refers to faster reaction times for larger numbers with right-sided responses and for smaller numbers with left-sided responses, even when numerical magnitude is irrelevant. Although the SNARC is generally thought to reflect a mapping between numbers and space, the question of which spatial reference frame(s) are critical for the effect has not been systematically explored. We propose a dynamic hierarchical organization of the reference frames (from a global left-right frame to body- and object-related frames), where the influence of each frame can be modulated by experimental context. We conducted two experiments based on predictions derived from this organizational system. Experiment 1 compared instructions that differed only in focusing participants' attention on either the response buttons or the hands. Instructions focusing on a hand-based reference frame eliminated the SNARC. Experiment 2 provided the opportunity for an object-centred reference frame to manifest itself in the SNARC. Although we did not observe an effect of an object-centred reference frame, we observed the influence of other reference frames in a context where an object-centred reference frame was emphasized. Altogether, these results support the proposed organization of the reference frames.
\end{abstract}

Keywords: Numerical cognition; Spatial reference frames; Spatial-numerical association of response codes effect.

Investigations of numerical cognition have shown that numbers are systematically mapped onto spatial locations: Smaller numbers are associated with the left side of space and larger numbers with the right side of space in Western participants who write from left to right (for reviews, see Fias \& Fischer, 2005; Hubbard, Piazza, Pinel, \& Dehaene, 2005; Wood, Willmes, Nuerk, \& Fischer, 2008). The earliest demonstration of this mapping is the spatial-numerical association of response codes (SNARC) effect, which is an association between numerical magnitude and the side of space on which participants respond when they make parity judgements (Dehaene, Bossini, \& Giraux, 1993). Responses to small numbers (relative to the range used in the experiment) are faster on the left side of space, whereas responses to large numbers are faster on the right. This effect has been replicated in multiple studies (see Wood et al., 2008, for a meta-analysis of 46 studies investigating the SNARC effect), and several characteristics have been reliably identified (automaticity, dependence on the experimental numerical interval, increasing magnitude with longer response times; see Gevers,

Correspondence should be addressed to Arnaud Viarouge, Laboratoire Psychologie de la Perception (CNRS, UMR8242), Université Paris Descartes, 45 Rue des Saints-Pères, 75006 Paris, France. E-mail: arnaud.viarouge@parisdescartes.fr 
Verguts, Reynvoet, Caessens, \& Fias, 2006, for a computational model that can account for these characteristics).

The different studies investigating the SNARC effect have revealed a range of experimental contexts in which the number-space association could be observed. In the field of spatial cognition, the characterization of the spatial reference frames (for example, self- vs. world-centred) is crucial. Identifying which of these reference frames is implicated in the SNARC effect is critical to understand the mechanisms that give rise to number-space associations. While the SNARC effect has generally been interpreted in terms of a spatial representation of numbers along a spatially oriented mental number line, others have argued that number-space associations result instead from the spatial ordering of numbers in working memory (Gevers et al., 2010; Van Dijck \& Fias, 2011). Independent of whether the SNARC effect reflects the spatial nature of numerical representation, the question remains of which reference frames are involved in the association of numbers to spatial codes. Previous work sheds light on the possible role of specific spatial reference frames in eliciting the SNARC effect. One of the best established distinctions in spatial cognition is that between reference frames centred on the self (egocentric) and centred on the external world (allocentric). Our question therefore becomes: Does the SNARC effect arise because of the position of the keys relative to the participant or does the reference frame act in a more global way? In the case of a reference frame centred on the self, the hands, the eyes, the body, or the head could each be the origin. Although these possibilities still need to be tested more thoroughly, several studies have recently indicated the possible role of a variety of reference frames, sometimes contrasting it with the role played by a more general leftright-oriented reference frame.

\section{Hand-based reference frames}

The hypothesis that the SNARC arises from a hand-based reference frame was first tested by asking participants to respond with either crossed or uncrossed hands (Dehaene et al., 1993). Using this method, Dehaene et al. (1993) found no effect of crossing the hands on the SNARC effect, arguing against the role of a hand-based frame. The same result was obtained in an experiment conducted by Müller and Schwarz (2007, Experiment 3), even when the experimental design emphasized a hand-based reference frame by keeping the assignment between hands and parity constant during the experiment. However, in the same study, when the response buttons were vertically arranged, and the emphasis again was placed on the hands, a hand-related SNARC effect (large numbers associated with the right hand, small numbers with the left hand) was observed, showing that a hand-based reference frame can be involved in the SNARC effect at least in some experimental conditions.

Other recent results have also demonstrated the possible role of a hand-based reference frame, even when the response buttons are arranged horizontally. Wood, Nuerk, and Willmes (2006) found that the SNARC effect was significantly reduced in the crossed-hands condition, suggesting a nonnegligible contribution of a hand-centred reference frame. They also discuss methodological differences between several studies investigating the SNARC effect with crossed and uncrossed hands. Notably, visual control over hands has a significant effect on the results obtained for these tasks, as in Fischer and Hill (2004) where the SNARC effect is significant only in the crossed-hands conditions when the visual control over hands is prevented. Finally, it is worth noting that a significant SNARC effect was previously found with pedal responses, which could also be interpreted as demonstrating an influence of a body-centred, effector-based reference frame (Schwarz \& Müller, 2006).

\section{Finger-based reference frames}

Building on the theory that finger counting may be the origin of the SNARC effect (see Wood \& Fischer, 2008, for a description of the theory of "manumerical cognition"), several recent studies have investigated the possible influence of a 
finger-based reference frame. For example, the SNARC effect can be influenced by counting habits (Fischer, 2008), or canonical hand representation of numbers between cultures (Domahs, Moeller, Huber, Willmes, \& Nuerk, 2010). Moreover, a recent study showed that a fingerbased reference frame could interact with the SNARC effect. In a parity task where subjects were asked to respond with the index and middle fingers of either their right or their left hand, Riello and Rusconi (2011) observed that unimanual SNARC effects were modulated by participants' hands position. When participants' hands were placed palm down, a unimanual SNARC effect was observed with the right hand, whereas a unimanual SNARC effect with the left hand was observed when the hands were palm up. This result, as well as other studies reporting a SNARC effect in the context of a parity task performed using two fingers of the same hand (Priftis, Zorzi, Meneghello, Marenzi, \& Umiltà, 2006), suggest that a finger-based reference frame can be relevant for eliciting a SNARC effect. However, in a tactile detection paradigm where short pulses were applied to the fingers of the right hand, the presentation of a small number facilitated the detection of a tactile stimulus delivered to the leftmost finger, and the presentation of a large number facilitated the detection of the stimulus delivered to the rightmost finger, independent of the position of the hand (Brozzoli et al., 2008). This result suggests a stronger involvement of a left-right-oriented (allocentric or egocentric) reference frame over a finger-based reference frame in the SNARC effect.

\section{Eye-centred reference frames}

Several studies have demonstrated the role of eyes in the numerical-spatial associations (Schwarz \& Keus, 2004). Fischer, Warlop, Hill, and Fias (2004) showed a SNARC effect using eye movement responses, and Loetscher, Bockisch, Nicholls, and Brugger (2010) showed that eye movements could predict the next number generated by participants in a random number generation task. One possible interpretation of these findings is that the SNARC effect may be due to shifts of attention within an eye-centred reference frame (Hubbard et al., 2005). As attention can be directed towards multiple aspects of space, including allocentric space, hands, fingers, and even foot locations, this suggestion would make it difficult to identify a single reference frame that is uniquely responsible for the SNARC effect.

\section{Object-centred reference frames}

Usually described as an example of an allocentric reference frame, the object-centred reference frame has been mostly investigated in studies of spatial neglect. Indeed, in addition to the core visual neglect, some patients also show additional deficits that can be interpreted as neglect in an object-centred reference frame. Patients showing this deficit ignore the left side of the presented object in, for example, line bisection tasks (Egly, Driver, \& Rafal, 1994; Karnath \& Rorden, 2012).

Operationally, it is at least possible that the magnitude of the SNARC effect would depend on the way in which the response buttons are centred on the object used to respond (e.g., response box, keyboard, etc.). To date, no study has investigated whether an object-centred reference frame is implicated in the SNARC effect.

The studies reviewed above suggest that several reference frames, including body-related ones, could play a role in eliciting an association between numbers and space. A global left-rightoriented reference frame seems to be predominantly involved in this association, although the question remains whether this reference frame is ego- or allocentric (see Conson, Mazzarella, \& Trojano, 2009, where an advantage for an egocentric reference frame is observed in the context of a hand laterality judgement task). While these studies point towards the possible role of many reference frames depending on the experimental context, one caveat of most of the SNARC studies is that these spatial reference frames are usually confounded (i.e., they are congruent with each other). For example, several of the effects reviewed above are also consistent with the 
hypothesis of an object-centred reference frame (considering the response set as the object of reference).

The general goal of the present study is to begin a systematic investigation into the reference frames involved in the SNARC effect. For that purpose, we propose an organizational system where the influence of the reference frames relative to one another are dynamically modified by the experimental context, rather than predetermined.

\section{A dynamic hierarchy of reference frames}

Based on the evidence reviewed above, it seems unlikely that only one system of coordinates causes the SNARC. Previous studies have already demonstrated that the way the stimuli are presented influences the reference frames involved in the SNARC effect. Bächtold, Baumuller, and Brugger (1998) found a reverse SNARC effect (left-hand advantage for large numbers) when participants were primed to imagine the numbers 1 to 11 as being positioned on a clock-face, whereas they replicated the standard SNARC effect when they asked participants to conceive these same numbers as being positioned on a ruler. Hung, Hung, Tzeng, and Wu (2008) found that, in Chinese participants, the direction of the SNARC effect could be modified by the notation of the stimuli, from a left-to-right orientation for Arabic numerals, to a top-to-bottom orientation for Chinese number words.

It thus seems reasonable to postulate the existence of a specific organizational system for the reference frames that can elicit number-space interactions. It is likely that several reference frames combine and/or compete to elicit the SNARC. Behavioural and brain imaging studies have pointed towards a hierarchical organization of the spatial reference frames, with the activation of allocentric representations being dependent on the one of egocentric representations (Pellizzer, Bâ, Zanello, \& Merlo, 2009; Zaehle et al., 2007). In a single neuron recording study conducted in monkeys, Crowe and colleagues reported the parietal area $7 \mathrm{a}$ as the potential locus for the transformation from a retina-centred to an object-centred representation (Crowe, Averbeck, \& Chafee, 2008). Thus, we suggest a hierarchical organizational system, in which the levels are defined as a function of the automaticity with which each reference frame can be involved in eliciting a SNARC effect. However, this hierarchy would dynamically evolve depending on the experimental context. Indeed, we predict dynamic shifts in relevance and therefore automaticity of the different reference frames depending on which frame has been most strongly activated by the experimental context.

Within this framework, the global left-right component of the SNARC effect, systematically observed in the most common experimental contexts, seems to be a predominant reference frame for the number-space association. Although it is unclear whether this left-right dimension is egoor allocentric, studies have shown that it resists experimental manipulation, such as crossing the hands (Dehaene et al., 1993), leading some authors to talk about the left-to-right-oriented mental number line as a reference frame in itself (Wood et al., 2008).

As described above, other studies have pointed towards the possible role played by reference frames that are more tightly linked to body parts or the specific effectors of the task, such as handbased, finger-based, or eye-centred reference frames (Brozzoli et al., 2008; Conson et al., 2009; Schwarz \& Keus, 2004). We suggest that these body-related reference frames constitute a second class of potential reference frames for the SNARC effect, potentially involved in numberspace association when emphasized by the experimental design.

Finally, following the organization described above, we suggest that a third group might consist in object-centred reference frames emerging from the experimental conditions themselves, such as a reference frame linked to the specific response set. In studies investigating the neural correlates of spatial reference frames, the parietal cortex, also known for its important role in numerical and spatial cognition, has been described as a location of overlapping neural correlates for both egocentric and allocentric reference frames (Committeri et al., 2004). Together with the presence of object-based 
neglect in patients with lesion in the parietal areas, these studies suggest that object-centred frames of reference could also be involved in the numberspace associations.

The general principles that we propose for this organizational system can be described as follows:

First, we suggest that the context in which the participant performs the task will spontaneously activate a preferred reference frame. This context corresponds to the general set-up of the task (response set, spatial location of the responses, visual feedback on the response set). For example, the studies reviewed above suggest that a global left-right reference frame is preferentially activated in the context of a task performed with two horizontally oriented response buttons located in front of the participant.

Next, we predict that other manipulations of the experimental design can activate secondary reference frames that will potentially interfere with the preferentially activated one. For example, the experimental design (block order ...) or the instructions can elicit such activation of one or several secondary reference frames. A recent study has shown that instructed stimulus-response associations elicited increased activation in several brain areas, including the intraparietal sulcus (Hartstra, Kühn, Verguts, \& Brass, 2011). These results, together with studies implicating the intraparietal region in number-space associations (Hubbard et al., 2005), suggest that manipulating the task instructions could have a direct impact on the observed SNARC effect. The impact on the magnitude of the observed SNARC effect will depend on how strongly the secondary reference frames are activated by these manipulations. One hypothesis is that the influence of these secondary frames depends on their congruency with the main one. If this hypothesis is correct, then in the crossedhand experiment, the effect of the hand-based reference frame is subtracted from the effects of the global left-right reference frame, leading to a weaker SNARC effect. Another hypothesis is that the activation of secondary reference frames requires recoding the association between the responses and the spatial codes of the currently evoked reference frame to another frame. The cost of this recoding could alter the magnitude of the observed SNARC effect even in contexts where the multiple reference frames evoked are congruent.

\section{The current study}

From this organizational system, we derive several predictions regarding the impact of manipulations of the experimental design on the magnitude of the SNARC effect in specific contexts.

For example, the question remains open whether some specific experimental conditions can lead to a hand-related SNARC effect even in the case where the response set is aligned with the left-right global reference frame. Indeed, a left-right SNARC effect is still observed in crossed-hands paradigms where the hand-based and the left-right reference frames are incongruent (Dehaene et al., 1993). We suggest that the impact of the hand-based (secondary) reference frame was not observed because its activation by the experimental context was not strong enough to interfere with the automatically activated left-right reference frame. Our hypothesis is that a variation of the instructions could affect the strength of the reference frames activated in a SNARC task. We predict that emphasizing the hands in the instructions will strengthen the hand-based reference frame and thus interfere with the preferentially activated left-right reference frame in the context of a parity task with horizontally aligned response buttons.

Assuming that the instructions impact the strength of reference frames, such experimental manipulations could then be adapted to try to observe number-space associations in less automatic (e.g., object-based) reference frames. Our proposed organizational system predicts that performing the parity task with vertically arranged response buttons will suppress the automatic activation of the left-right reference frame. Then, manipulation of the experimental design and instructions could strengthen the activation of an object-reference frame, which in turn will lead to an association between numbers and the sides of the object. 
VIAROUGE, HUBBARD, DEHAENE

\section{EXPERIMENT 1: INSTRUCTIONAL EFFECTS ON THE SNARC}

This first experiment was aimed at testing whether manipulating the instructions could strengthen the activation of a secondary, hand-based, reference frame in the context of a parity judgement task with horizontally arranged response buttons. Two groups of participants performed the same parity judgement task with different instructions. The first set of instructions stressed the parity-tobutton assignments, while the second set of instructions stressed the parity-to-hand assignments. Each group performed the task with crossed and uncrossed hands, which allowed us to explore the relative contributions of hand-based and more global, left-right-oriented, reference frames. Our proposed organizational system for the reference frames predicts that, in the case of button-based instructions, a global left-right reference frame should be preferentially activated, leading to a classic left-right SNARC effect in both uncrossed- and crossed-hands conditions. However, we expected that this pattern would be modified by instructions focusing on hands. Indeed, our model predicts that such manipulation should strengthen the activation of a hand-based reference frame, impacting the magnitude of the observed SNARC effect. If this impact depends on the congruency between the reference frames, we should observe a weaker SNARC effect in the crossed-hands condition, and a similar or stronger SNARC effect in the uncrossed-hands condition. However, if the observed SNARC effect is affected by the cost of recoding the association between responses and the activated reference frames, a weaker SNARC effect should be observed in both the crossed- and the uncrossed-hands conditions.

\section{Method}

\section{Participants}

A total of 48 French participants (mean age 22.6 years) gave informed consent before taking part in this experiment and received compensation of 5 euros (approximately \$7) for their participation.
Participants were all right-handed according to the Edinburgh Handedness Inventory (Oldfield, 1971) and had normal or corrected-to-normal vision. None of the participants was familiar with the hypotheses of this study.

\section{Stimuli and procedure}

The experiment was programmed using E-prime software on a HP/Compaq 1.7-GHz portable personal computer. Stimuli were Arabic digits between 1 and 9, excluding 5, presented in Courier New, size 18 , which corresponded to a $0.42 \times 1.15-\mathrm{cm}$ surface on the screen. Thus, the visual eccentricity of the stimuli was $0.48^{\circ}$ horizontally and $1.32^{\circ}$ vertically. For each trial, a fixation cross appeared at the centre of the screen for $200 \mathrm{~ms}$, followed by the target digit for 1600 ms. Feedback was then presented for $600 \mathrm{~ms}$ at the top of the screen, indicating the response time in a different colour depending on whether the answer was correct or not (blue for a correct answer, red for an incorrect answer, green if no answer was recorded). The participants were instructed to ignore this feedback unless they forgot or became confused about the stimulus-response mapping during the experiment, in order to keep their concentration on the centre of the screen. The distance between the participants and the screen was approximately $60 \mathrm{~cm}$. The participants responded with the index fingers of each hand by pressing two independent response buttons, placed in front of the keyboard and separated by a fixed distance of $16 \mathrm{~cm}$.

For 24 participants, the instructions focused on their hands, while for the other 24 participants, the instructions focused on the response buttons. In each group, the participants performed two blocks with one hand position (cross/uncrossed) and then two with the other, counterbalanced between participants. For each hand position, two blocks were performed corresponding to the two possible assignments of parity to hands/buttons (depending on the instructions). Thus, in the "handsinstruction" group, the participants were given a parity-to-hands assignment, whereas in the "buttons-instruction" group they were given a parity-to-buttons assignment (see Appendix for exact wording of the instructions). Each block 
was preceded by eight practice trials (one for each digit, in random order) followed immediately by 20 presentations of each digit in a random order. The task therefore consisted of four blocks of 160 trials for a total of 640 trials. The entire experiment took approximately 40 minutes.

\section{Results}

Error rates were less than $15 \%$ for all participants (mean error rate $=4.58 \%, S D=2.71 \%$ ). Only correct trials with reaction times (RTs) between 150 and $1200 \mathrm{~ms}$ were further analysed. We performed two analyses to test for the presence and magnitude of the SNARC effect. First, we computed mean RTs for each participant and each experimental condition and then performed a 2 (instructions: hands/buttons) $\times 2$ (buttons: left/ right $) \times 2$ (hands: left/right) $\times 2$ (magnitude: small/large) mixed analysis of variance (ANOVA), with response buttons, hands, and magnitude as within-participants factors and instructions as a between-participants factor. Second, we calculated two measures of the difference in RTs ("dRT") for each participant, relative to the response buttons $[\mathrm{dRT}$ (buttons) $=$ mean RT for right button - mean RT for left button] and relative to the hands $[\mathrm{dRT}$ (hands) $=$ mean $\mathrm{RT}$ for the right hand - mean RT for the left hand]. We then used these dRTs to calculate the regression slope for each participant (Fias, Brysbaert, Geypens, \& D’Ydevalle, 1996; Lorch \& Myers, 1990). T-tests were then computed on the mean regression slopes obtained for each experimental condition. A negative slope indicates a SNARC effect linked either to the response buttons or to the hands.

First, the ANOVA showed a significant main effect of response hand, $F(1,46)=18.73$, $p<.001$, revealing a global advantage for righthand responses $(506 \mathrm{~ms}$ for right-hand responses vs. $516 \mathrm{~ms}$ for left-hand responses, collapsed across hand positions), consistent with the fact that all participants were right-handed. We also observed a global advantage for left-button responses compared to right-button responses, $F(1, \quad 46)=4.26, \quad p=.04$. The significant
Hands $\times$ Button interaction, $F(1,46)=31.96$, $p<.001$, indicates a global advantage for responses given with uncrossed hands (that is, when the position of the hands is congruent with the position of the buttons, $497 \mathrm{~ms}$ uncrossed vs. $526 \mathrm{~ms}$ crossed).

More importantly, we observed a global SNARC effect relative to the buttons, as demonstrated by a significant Magnitude (small: 1, 2, 3, 4; vs. large: $6,7,8,9) \times$ Response Button interaction, $F(1,46)=22.77, p<.001$. The absence of a significant Magnitude $\times$ Hands interaction, $F(1,46)=0.84, p=.36$, indicates that, across both instructions, the global left-right reference frame played a greater role in the SNARC effect than did the hand-centred reference frame. However, we also observed a marginally significant Instructions $\times$ Magnitude $\times$ Hands interaction, $F(1,46)=3.99, p=.052$, suggesting that the instructions were effective in leading participants to focus on the hand-centred reference frame. This interaction becomes significant when magnitude is treated as a continuous variable, $F(1,46)=4.87, p=.03$.

In order to test our predictions regarding the effects of the instructional changes on the uncrossed- and crossed-hands conditions, we examined the effect of hand position (crossed or uncrossed) for each instruction set by computing separate ANOVAs for each instruction set. Figure 1 shows the two types of analyses (interaction and $\mathrm{dRT}$ ) computed relative to the position of the buttons, in the crossed- and uncrossed-hands conditions for both types of instructions.

\section{Buttons instructions}

For the group who received the instructions stressing the parity-to-buttons assignment, we observed a SNARC effect linked to the side of the response button when the hands were uncrossed, with a significant Magnitude $\times$ Response Button interaction, both when magnitude was treated as a categorical variable, $F(1,23)=18.51, p<.001$, and when it was treated as a continuous variable, $F(1,23)=$ 14.99, $p<.001$. The regression slope computed from the dRTs relative to the buttons was significantly negative (slope $=-5.89, p<.001$ ), confirming the presence of a SNARC effect in this 
VIAROUGE, HUBBARD, DEHAENE
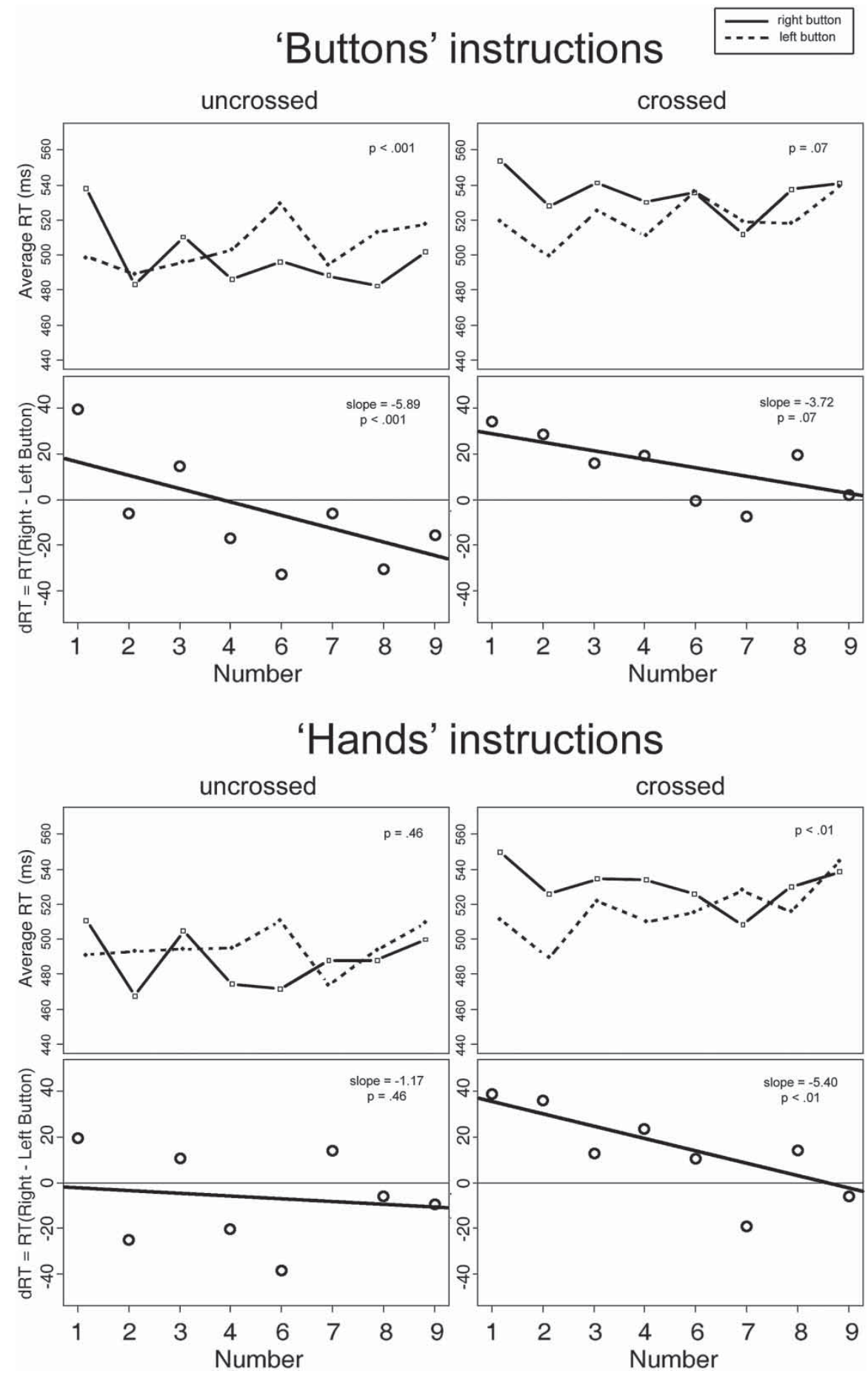

Figure 1. Average reaction times and regression lines in the "uncrossed hands" (left-side graphs) and "crossed hands" (right-side graphs) conditions, for each instructional condition. $R T=$ reaction time (in $m s$ ); $d R T=$ difference in $R T s$ (in $m s$ ). 
condition. In the hands-crossed condition, although the Magnitude $\times$ Response Button interaction was not significant when treated as a categorical variable, $F(1,23)=3.67, p=.07$, it was significant when magnitude was treated as a continuous variable, $F(1,23)=5.03, p=.03$. The regression analysis also showed a nonsignificant SNARC effect linked to the side of the buttons (slope $=-3.72, p=.07$ ). Additionally, the Magnitude $\times$ Response Button $\times$ Hands Position ANOVA over the entire "buttons instruction" group did not indicate any interaction between the SNARC effect and the position of hands, $F(1,23)=0.44, p=.51$ (also when using numbers instead of the magnitude). This result confirms that the SNARC effect is linked to response side, independently of the position of the hands, in agreement with previous results (Dehaene et al., 1993).

\section{Hands instructions}

Unlike in the "buttons instructions" group, a significant interaction between the position of the hands and the SNARC effect was found in the "hand instruction" group (Hands $\times$ Magnitude $\times$ Response Buttons interaction), both when we treated magnitude as a categorical, $F(1,23)=5.90$, $p=.02$, and when we treated it as a continuous, $F(1,23)=6.19, p=.02$, variable. Indeed, the SNARC effect was significant in the crossedhands condition $[F(1,23)=11.24, p<.01$, categorical; $F(1,23)=11.33, p<.01$, continuous], with a negative slope for the regression analysis on dRTs $(\mathrm{dRT}=-5.40 \times x+40.81, p<.01)$, but not in the uncrossed-hands condition $[F(1,23)=$ $0.57, \quad p=.46, \quad$ continuous; $\quad \mathrm{dRT}=-$ $1.17 \times x-1.20, p=.46]$.

A 2 (instructions: buttons or hands) $\times 2$ (magnitude) $\times 2$ (response buttons) analysis performed on the entire set of RTs confirmed the effect of instructions on the SNARC effect in the uncrossed-hands condition $[F(1,44)=4.57$, $p=.04$, categorical; $F(1,44)=5.05, p=.03$, continuous]. The same analysis performed in the crossed-hands condition did not show an effect of the instructions on the SNARC effect $[F(1$, $44)=0.3, p=.59$, categorical; $F(1,44=0.43$, $p=.51$, continuous], but on the contrary showed a very strong global SNARC effect $[F(1,44)=$ 14.79, $p<.001$, categorical; $F(1,44)=12.75$, $p<.001$, continuous]. These results indicate that our instructions had an effect when participants were in the standard uncrossed-hands condition. On the other hand, in the crossed-hands condition, the absence of an effect suggests that the dominant spatial reference frame is independent of the hands.

\section{EXPERIMENT 2: OBJECT-CENTRED REFERENCE FRAME}

Experiment 1 suggests that the instructions given to the participant can affect the magnitude of the SNARC effect by modulating the role of a secondary, hand-based reference frame, in a context where a global left-right reference frame is dominant. Due to the horizontal arrangement of the buttons, this global reference frame could have been either allocentric or body centred. However, it could also have been centred on the buttons themselves. To investigate the role of an object-based reference frame, we set up a protocol using buttons placed into an object with a clear axis of symmetry, which allowed us to disentangle the influence of an object-based reference frame from that of other reference frames. Our organizational system predicts that the context of a parity task with vertically arranged response buttons would minimize the influence of a left-right-oriented reference frame. This experimental context would then allow other reference frames, such as a hand-based or an object-based frame, to exert an influence on the SNARC effect. Following the predictions of the hierarchy of reference frames and given that Experiment 1 had already demonstrated that the instructions had an effect on the SNARC, we hypothesized that focusing on the sides of the boat in the instructions would strengthen the influence of the reference frame based on the response set.

\section{Method}

\section{Participants}

A total of 16 new French participants (mean age 22.1 years) were recruited to participate in one 
Session 1

1)

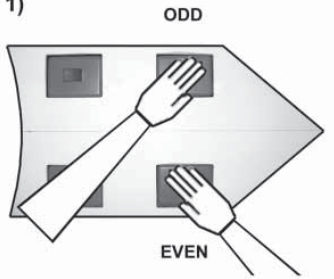

Session 2

1)

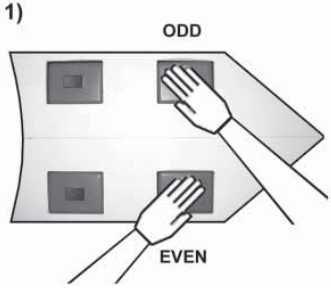

2)

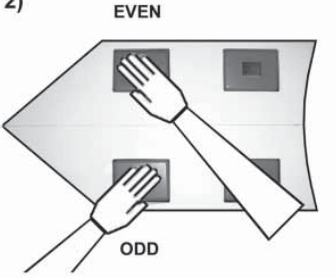

2)

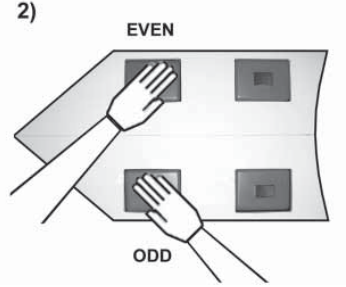

3)
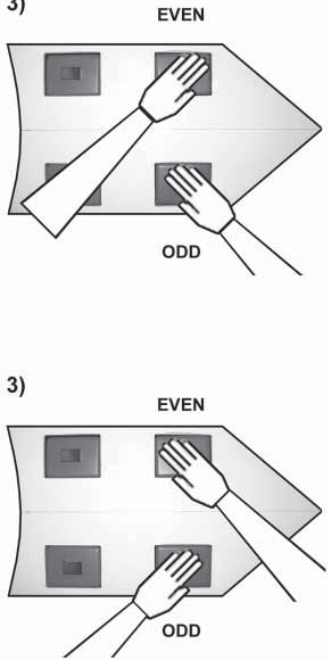
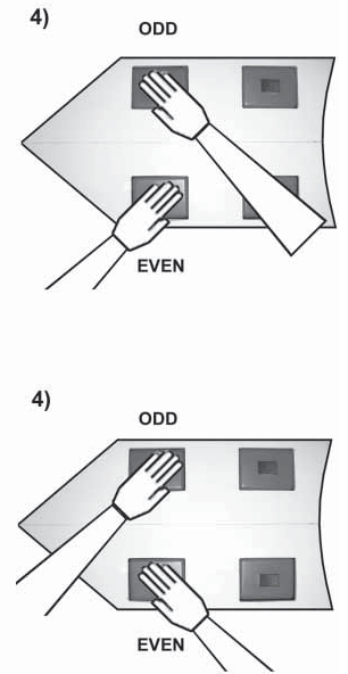

Figure 2. Schematic representation of the experimental design of Experiment 2, in one of the eight possible orders regarding the position of hands, the parity assignment, and the orientation of the "boat", across the four blocks of each of the two sessions.

session of 75 minutes (compensated 15 euros). All participants gave informed consent prior to beginning the experiment. They were all right-handed French native speakers with normal or correctedto-normal vision. Additionally, we verified that none of the participants had ever learned to write using a right-to-left writing system, as this has previously been shown to influence the SNARC effect (Dehaene et al., 1993; Zebian, 2005). None of the participants were familiar with the hypotheses of the study.

\section{Stimuli and procedure}

The same parity judgement task as that in Experiment 1 was used, with the response buttons located within an object that had an intrinsic front/back orientation (the shape of a boat) and was symmetric relative to the axis defining this orientation (bilateral symmetry, see Figure 2). The feedback screen was removed to optimize the participants' concentration on the centre of the screen. The response object was explicitly described to the participants as representing a boat. The experimenter indicated the orientation of the boat and the presence of two buttons on both sides of its deck (on the left/port and right/starboard sides of the boat). The boat could be oriented either $90^{\circ}$ to the right or $90^{\circ}$ to the left, which had the effect of positioning the two active buttons on a vertical axis, thus eliminating the left/right component linked to these two buttons. Depending on the orientation of the boat, the same button could correspond to either the right side or the left side of the response set. Participants made their responses by pressing two buttons on the front portion of the boat, on each side of its axis of symmetry. The experiment was divided into two sessions, performed one after the other, with a short break between them. Within each session, participants were told to keep their hands on the same side of the boat, independent of the orientation of the boat (left or right), which was changed after each block. The parity to boat side assignment was also maintained between two consecutive blocks. The order of the three variables (boat orientation, response side, and position of the hands) was determined by their starting value for the very first block of the experiment. These starting values were counterbalanced between participants, leading to eight possible orders. Figure 2 shows the experimental design for one of the eight possible orders, with a boat initially 

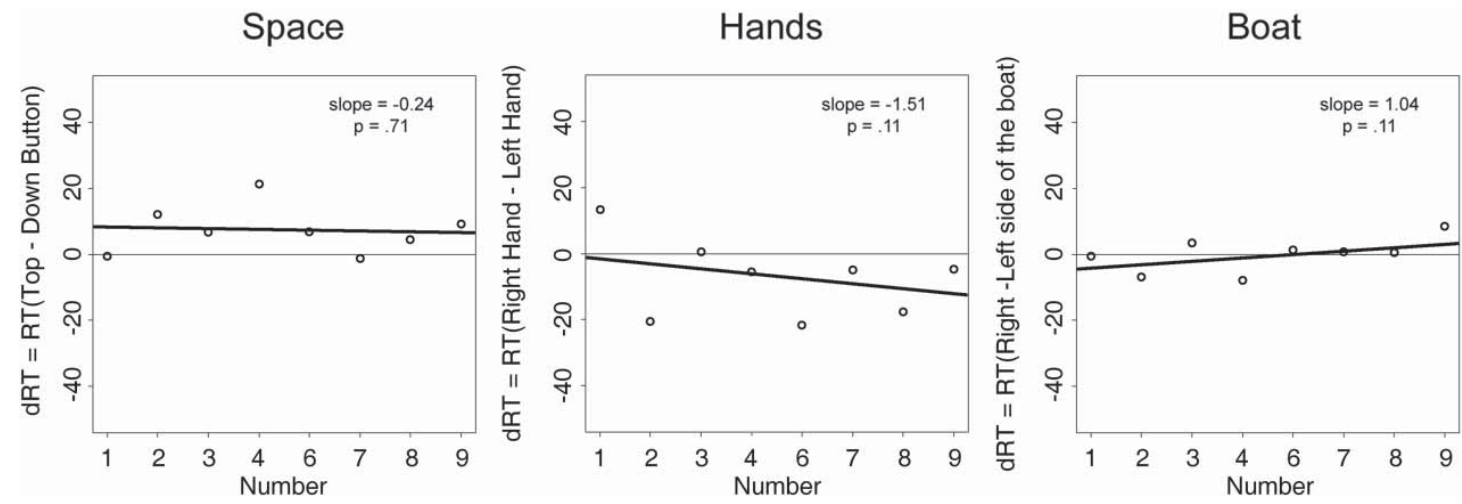

Figure 3. Regression lines obtained from the dRTs (in ms) calculated relatively to the response buttons ("space"), the hands, or the side of the "boat". $R T=$ reaction time; $d R T=$ difference in $R T s$.

oriented to the right, even numbers initially assigned to the bottom button, and the right hand initially placed at the bottom position. At the beginning of each block, the experimenter reminded participants of the place where the hands should stay and also indicated on which side of the boat the "odd" and "even" responses were to be given.

\section{Results}

Error rates were less than $10 \%$ for all participants (mean error rate $=4.88 \%, S D=2.67 \%$ ). As in Experiment 1, we excluded errors and RTs that were less than $150 \mathrm{~ms}$ or more than $1200 \mathrm{~ms}$. The 2 (hand: left/right) $\times 2$ (boat side: left/right) $\times 2$ (response button: top/bottom) $\times 2$ (magnitude: small/large) $\times 2$ (parity: odd/even) ANOVA showed global effects of hands [faster responses with the right hand, mean $491 \mathrm{~ms}$ vs. $499 \mathrm{~ms}$; $F(1,15)=8.24, p=.01]$, parity [faster responses to even numbers, mean $489 \mathrm{~ms}$ vs. $500 \mathrm{~ms}$; $F(1,15)=8.84, p<.01]$, and the response button [faster responses with the bottom button, mean $491 \mathrm{~ms}$ vs. $499 \mathrm{~ms} ; \quad F(1,15)=14.46$, $p<.01]$. Finally, we observed a significant Parity $\times$ Magnitude interaction, $F(1,15)=5.23$, $p=.04$.

Although we found significant main effects in this experiment, none of the three interactions between magnitude and each of the variables corresponding to a spatial reference frame was significant. Only the Magnitude $\times$ Hands interaction showed a trend towards significance in the expected direction regarding the SNARC effect, $F(1,15)=$ $4.5, p=.051$. Figure 3 shows the corresponding analysis in terms of regression slopes computed from the dRTs defined as in the previous experiment.

Although the slope of the regression on dRTs computed relative to the hands was negative $(\mathrm{dRT}=-1.51 \times x-0.10), t(15)=-1.7, p=.11$, the regression analysis on the slopes was not significant for any of the three dRT measures, suggesting the absence of a SNARC effect in the three tested reference frames. We observed no SNARC effect linked to the response buttons, $t(15)=-0.38, p=.71$, or to the side of the boat, $t(15)=1.67, p=.11$, for which the slope goes in the direction of a reversed SNARC effect. When we examined the dRTs relative to the position of the participants' hands, the slope of the regression computed from the RTs on the buttons was reversed when the participant's right hand was located on the bottom button $(\mathrm{dRT}=$ $1.22 \times x+8.88), t(15)=1.06, p=.30$, compared to when the right hand was located on the top button $(\mathrm{dRT}=-1.78 \times x+8.43), \quad t(15)=-$ $1.73, p=.10$. This was confirmed by a marginally significant triple interaction between magnitude, response button, and position of the hands, $F(1$, $15)=4.50, p=.05$. These results indicate that 

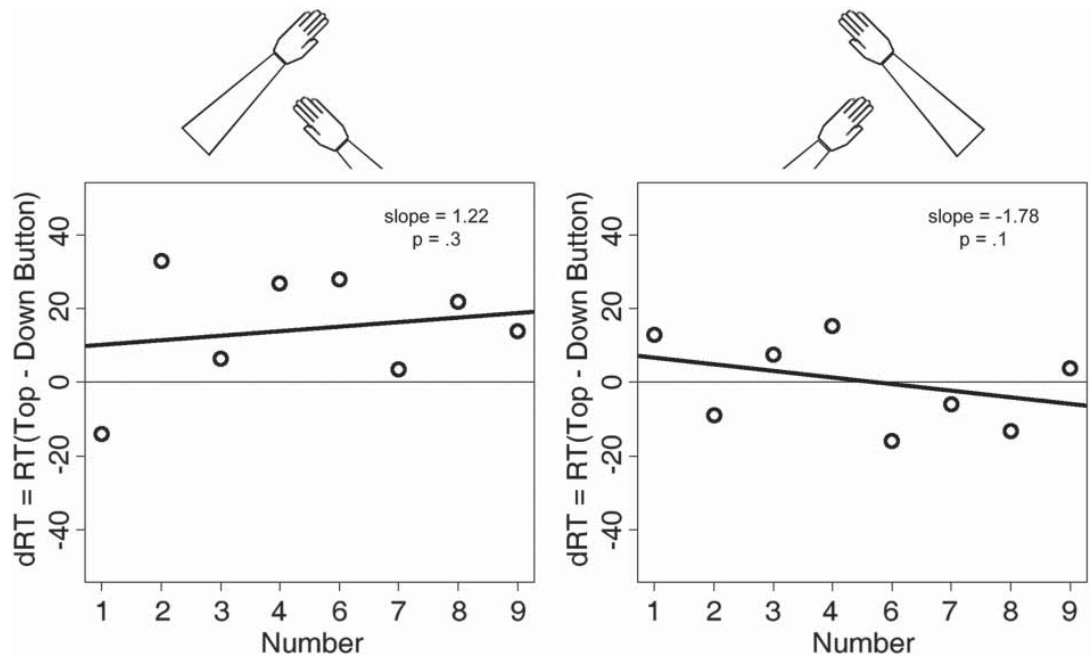

Figure 4. Regression analysis of the response-button-related dRTs (in ms), in the case where the right hand is the closest to the participant (left-side graph), and in the case where the left hand is the closest to the participant (right-side graph). $R T=$ reaction time; $d R T=$ difference in $R T$ s.

larger numbers were mainly associated with the right hand rather than with the top button in this condition (Figure 4).

\section{GENERAL DISCUSSION}

Many studies have investigated the links between numbers and space, particularly with respect to the automatic activation of a spatial representation observed when participants are presented with numerical stimuli. However, there are still many open questions regarding the precise characterization of the reference frames implicated in these numerical-spatial associations. By relying on classifications habitually used in the field of spatial cognition, we suggested a framework to investigate the role of several spatial reference frames in the SNARC effect. Following recent studies that have demonstrated that number-space mappings may be flexibly constructed, we proposed a dynamic, hierarchical organization of spatial reference frames that can interact and compete with each other, depending on the experimental context, so that the most relevant is chosen by the participant according to the instructions and yields the effects observed on reaction times.
While the number of reference frames and their combinations leads to a combinatorial explosion of possible experiments, we performed two experiments that provide a first attempt to systematically assess this organizational system of reference frames.

Experiment 1 allowed confirmation of two predictions of our model. The first one is the advantage of a global left-right-oriented reference frame in the context of a task performed with horizontally arranged response buttons. Participants who received instructions that focused them on the response buttons demonstrated a clear SNARC effect associated with the side of space, even when the participants performed the task with their hands crossed. The presence of a SNARC effect, both in the hands-normal and in the hands-crossed position indicates that a more global, effector-independent spatial reference frame dominated over a hand-centred reference frame, consistent with previous studies (Dehaene et al., 1993; Müller \& Schwarz, 2007).

The second prediction that was confirmed in this experiment is that changing the instructions can activate secondary reference frames, which in turn impacts the observed SNARC effect. In our experiment, while keeping all other aspects of the

\section{2}

THE QUARTERLY JOURNAL OF EXPERIMENTAL PSYCHOLOGY, 2014 
experimental design constant, giving the participants instructions that focused them on their hands significantly impacted on the magnitude of the SNARC effect. When looking at this result more closely we observed a surprising pattern. With instructions focusing on hands, when participants' hands were uncrossed, no SNARC effect was observed. However, when the same participants responded with their hands crossed, a significant classic left-right SNARC effect was observed.

This pattern of results suggests that the magnitude of the observed SNARC effect does not depend solely on the congruency between the activated reference frames. Indeed, the "uncrossed hands" condition should then have led to a stronger SNARC effect.

However, these results are consistent with the idea that manipulating the experimental context requires recoding the responses to a newly activated reference frame, which in turn affects the strength of the number-space association. That is, in the "uncrossed hands" condition, the recoding of responses to a hand-based reference frame may have weakened the SNARC effect. In the Müller and Schwarz (2007) experiment, which is the closest to our "hands instructions" condition, the condition where the participants had their hands uncrossed also yielded to a weaker SNARC effect. However, in the "crossed hands" condition, the parity-to-hand mapping may have been too difficult, leading the participants to discard the instructions and rely on canonical (left button/ right button) response codes. This condition led to a classic left-right SNARC effect.

A previous study failed to observe a hand-related SNARC effect when the buttons were oriented horizontally, even when using an experimental design that emphasized the hands (Müller \& Schwarz, 2007). Based on this, the authors concluded that the instructions did not modify the SNARC in the horizontal orientation, and that the use of a vertical orientation was necessary to reveal the subtle effects of instructions. We suggest that the authors may have been too hasty in concluding that there was no effect of the instructions in this configuration, since they did not directly test this effect using the exact same experimental design, as we did here. Despite this difference, our results generally agree with those obtained by Müller and Schwarz (2007), since we still observed an advantage of a global reference frame linked to the side of the responses, even when participants were given instructions focusing on hands. We thus come to the same conclusion concerning the predominance of the left-right dimension in the standard situation where responses are given on horizontally arranged buttons.

In Experiment 2, we tested a prediction of our organizational system according to which an object-based reference frame could be involved in the SNARC effect when this frame was emphasized by the experimental context. The parity task was performed with vertically arranged buttons, which suppressed the influence of the canonical left-right-oriented reference frame. In a similar context, several studies have shown an advantage for a hand-based reference frame (Ito \& Hatta, 2004; Shaki \& Fischer, 2012), especially when the experimental design favours the hand-parity assignment (Müller \& Schwarz, 2007). Consistently with those prior results, our results show a trend towards an advantage of a handbased reference frame over the reference frame linked to the bottom-top dimension.

In our experiment, however, we added the possible influence of a reference frame linked to the response set. Even though we did not observe a SNARC effect based on this reference frame, the experimental design and the instructions weakened (and cancelled) the influence of the two other reference frames (linked to the bottom-top dimension and linked to the hands). Thus, our results suggest that the manipulations of the experimental design did activate an additional object-based, reference frame. Further studies are needed to investigate the number-space mappings in objectcentred reference frames more specifically. For instance, it is possible that the block sequence in Experiment 2 could still be further modified to optimize the recognition of this reference frame by the participants. In the design of Experiment 2 , the assignment between parity and the side of the object was maintained during the same 
number of blocks as that for the hand-parity assignment. The activation of the object-based reference frame could possibly have been strengthened by maintaining the parity to boat-side mapping during four consecutive blocks. Participants often reported having difficulties following the instructions (which asked them to think about the responses in terms of sides of the boat), and the change in the block sequence may have contributed to the difficulty in coding the responses into an object-based reference frame.

Overall, our results support the hypothesis that the SNARC effect is linked to several distinct spatial reference frames, whose influence can be modulated depending on the experimental context, and in particular according to the ease with which the participant can follow the assignment between the response and these different reference frames. The mental number line (MNL), which is thought to be a left-to-right organization of the integers along a horizontal axis, is often referred to as a specific reference frame (Wood et al., 2006). Assuming the existence of this internal reference frame for the MNL, Wood et al. (2006) explain their results in terms of a congruence or incongruence between the reference frame for the MNL and the other frames available in the context of the experiment.

From a developmental point of view, we suggest that at the time when children first learn the sequence of integers, the spatial reference frames we have described are highly correlated (as children normally sit facing more or less straight ahead in a classroom setting) and thus play an equal role in the development of the space-number association. Several studies have examined the development of spatial reference frames in young children, notably showing that the retinocentric reference frame develops very early, and that egocentric reference frames may become available in 4-month-old infants (Kaufman, Gilmore, \& Johnson, 2006). Thus it seems plausible to argue that children already have all of the relevant reference frames in place when they begin education and first are taught mathematics.

We therefore suggest that this hypothesis of a unique reference frame for the mental number line is unnecessary. Rather, we argue that the presentation of a numerical stimulus calls up one of the multiple preexisting spatial reference frames in which the numbers can be placed, depending on the context of the experiment. Altogether, our results support the hypothesis that numbers can be flexibly mapped onto different reference frames, depending on context, instructions, and other factors, including cultural traditions like the direction of reading and writing. Additionally, we believe the framework proposed here may provide a powerful organizing structure that can help make sense of the literature and furthermore motivate and guide future research.

Original manuscript received 15 July 2013
Accepted revision received 24 January 2014
First published online 19 March 2014

\section{REFERENCES}

Bächtold, D., Baumuller, M., \& Brugger, P. (1998). Stimulus-response compatibility in representational space. Neuropsychologia, 36(8), 731-735.

Brozzoli, C., Ishihara, M., Göbel, S. M., Salemme, R., Rossetti, Y., \& Farnè, A. (2008). Touch perception reveals the dominance of spatial over digital representation of numbers. Proceedings of the National Academy of Sciences, 105(14), 5644-5648.

Committeri, G., Galati, G., Paradis, A.-L., Pizzamiglio, L., Berthoz, A., \& LeBihan, D. (2004). Reference frames for spatial cognition: Different brain areas are involved in viewer-, object-, and landmark-centered judgments about object location. Journal of Cognitive Neuroscience, 16(9), 1517-1535.

Conson, M., Mazzarella, E., \& Trojano, L. (2009). Numbers are represented in egocentric space: Effects of numerical cues and spatial reference frames on hand laterality judgements. Neuroscience Letters, 452(2), 176-180.

Crowe, D. A., Averbeck, B. B., \& Chafee, M. V. (2008). Neural ensemble decoding reveals a correlate of viewer- to object-centered spatial transformation in monkey parietal cortex. The Journal of Neuroscience: The Official Journal of the Society for Neuroscience, 28 (20), 5218-5228.

Dehaene, S., Bossini, S., \& Giraux, P. (1993). The mental representation of parity and number

\section{THE QUARTERLY JOURNAL OF EXPERIMENTAL PSYCHOLOGY, 2014}


magnitude. Journal of Experimental Psychology: General, 122, 371-396.

Domahs, F., Moeller, K., Huber, S., Willmes, K., \& Nuerk, H.-C. (2010). Embodied numerosity: Implicit hand-based representations influence symbolic number processing across cultures. Cognition, 116(2), 251-266.

Egly, R., Driver, J., \& Rafal, R. D. (1994). Shifting visual attention between objects and locations: Evidence from normal and parietal lesion subjects. Journal of Experimental Psychology: General, 123(2), 161-177.

Fias, W., Brysbaert, M., Geypens, F., \& D’Ydevalle, G. (1996). The importance of magnitude information in numerical processing: Evidence from the SNARCeffect. Mathematical Cognition, 2, 95-110.

Fias, W., \& Fischer, M. H. (2005). Spatial representation of numbers. In J. I. D. Campbell (Ed.), Handbook of mathematical cognition (pp. 43-54). Hove: Psychology Press.

Fischer, M. H. (2008). Finger counting habits modulate spatial-numerical associations. Cortex, 44(4), 386392.

Fischer, M. H., \& Hill, R. L. (2004, January 25-30). A SNARC in the dark. 22st Workshop on Cognitive Neuropsychology, Bressanone, Italy.

Fischer, M. H., Warlop, N., Hill, R. L., \& Fias, W. (2004). Oculomotor bias induced by number perception. Experimental Psychology, 51(2), 91-97.

Gevers, W., Santens, S., Dhooge, E., Chen, Q., Van den Bossche, L., Fias, W., \& Verguts, T. (2010). Verbalspatial and visuospatial coding of number-space interactions. Journal of Experimental Psychology: General, 139(1), 180-190.

Gevers, W., Verguts, T., Reynvoet, B., Caessens, B., \& Fias, W. (2006). Numbers and space: A computational model of the SNARC effect. Journal of Experimental Psychology: Human Perception and Performance, 32(1), 32-44.

Hartstra, E., Kühn, S., Verguts, T., \& Brass, M. (2011). The implementation of verbal instructions: An $\mathrm{fMRI}$ study. Human Brain Mapping, 32(11), 1811-1824. doi:10.1002/hbm.21152

Hubbard, E. M., Piazza, M., Pinel, P., \& Dehaene, S. (2005). Interactions between number and space in parietal cortex. Nature Reviews Neuroscience, 6(6), 435-448.

Hung, Y.-hui, Hung, D. L., Tzeng, O. J.-L., \& Wu, D. H. (2008). Flexible spatial mapping of different notations of numbers in Chinese readers. Cognition, 106 (3), 1441-1450.
Ito, Y., \& Hatta, T. (2004). Spatial structure of quantitative representation of numbers: Evidence from the SNARC effect. Memory E Cognition, 32(4), 662673.

Karnath, H.-O., \& Rorden, C. (2012). The anatomy of spatial neglect. Neuropsychologia, 50(6), 1010-1017.

Kaufman, J., Gilmore, R. O., \& Johnson, M. H. (2006). Frames of reference for anticipatory action in 4month-old infants. Infant Behavior E Development, 29, 322-333.

Loetscher, T., Bockisch, C. J., Nicholls, M. E. R., \& Brugger, P. (2010). Eye position predicts what number you have in mind. Current Biology, 20(6), R264-R265.

Lorch, R. F., \& Myers, J. L. (1990). Regression analyses of repeated measures data in cognition research. Journal of Experimental Psychology: Learning, Memory, and Cognition, 16, 149-157.

Müller, D., \& Schwarz, W. (2007). Is there an internal association of numbers to hands? The task set influences the nature of the SNARC effect. Memory E० Cognition, 35(5), 1151-1161.

Oldfield, R. C. (1971). The assessment and analysis of handedness: The Edinburgh inventory. Neuropsychologia, 9, 97-113.

Pellizzer, G., Bâ, M. B., Zanello, A., \& Merlo, M. C. G. (2009). Asymmetric learning transfer between imagined viewer- and object-rotations: Evidence of a hierarchical organization of spatial reference frames. Brain and Cognition, 71(3), 272-278.

Priftis, K., Zorzi, M., Meneghello, F., Marenzi, R., \& Umiltà, C. (2006). Explicit versus implicit processing of representational space in Neglect: Dissociations in accessing the mental number line. Journal of Cognitive Neuroscience, 18(4), 680-688.

Riello, M., \& Rusconi, E. (2011). Unimanual SNARC effect: Hand matters. Frontiers in Psychology, 2, 372. doi:10.3389/fpsyg.2011.00372

Schwarz, W., \& Keus, I. M. (2004). Moving eyes along the mental number line: Comparing SNARC effects with saccadic and manual responses. Perception and Psychophysics, 66, 651-664.

Schwarz, W., \& Müller, D. (2006). Spatial associations in number-related tasks: A comparison of manual and pedal Responses. Experimental Psychology, 53 (1), 4-15.

Shaki, S., \& Fischer, M. H. (2012). Multiple spatial mappings in numerical cognition. Journal of Experimental Psychology: Human Perception and Performance, 38(3), 804-809. 
VIAROUGE, HUBBARD, DEHAENE

Van Dijck, J.-P., \& Fias, W. (2011). A working memory account for spatial-numerical associations. Cognition, 119(1), 114-119.

Wood, G., \& Fischer, M. H. (2008). Numbers, space, and action - From finger counting to the mental number line and beyond. Cortex, 44(4), 353-358.

Wood, G., Nuerk, H.-C., \& Willmes, K. (2006). Crossed hands and the SNARC effect: A failure to replicate Dehaene, Bossini and Giraux (1993). Cortex, 42(8), 1069-1079.

Wood, G., Willmes, K., Nuerk, H.-C., \& Fischer, M. (2008). On the cognitive link between space and

\section{APPENDIX}

\section{Verbatim instructions for Experiment 1}

Hand-based instructions (translated from French)

"You will be asked to respond as quickly as possible while making as few mistakes as possible by using your right hand for an even number and your left hand for an odd number."

\section{Button-based instruction}

"You will be asked to respond as quickly as possible while making as few mistakes as possible by using the right button for an even number and the left button for an odd number." number: A meta-analysis of the SNARC effect. Psychology Science Quarterly, 50(4), 489-525.

Zaehle, T., Jordan, K., Wüstenberg, T., Baudewig, J., Dechent, P., \& Mast, F. W. (2007). The neural basis of the egocentric and allocentric spatial frame of reference. Brain Research, 1137(1), 92-103.

Zebian, S. (2005). Linkages between number concepts, spatial thinking, and directionality of writing: The SNARC effect and the REVERSE SNARC effect in english and arabic monoliterates, biliterates, and illiterate arabic speakers. Journal of Cognition $\xi^{\circ}$ Culture, 5(1-2), 165-190.

\section{Verbatim instructions for Experiment 2}

"This object represents a boat. You can respond on the port, that is the right side of the boat (the experimenter shows the button located on the right side of the object), or on the starboard, that is the left side of the boat (the experimenter shows the button located on the left side of the object). In the following block, you will be asked to respond on the port, that is the right side of the boat for even (resp. odd) numbers, and on the starboard, that is the left side of the boat for odd (resp. even) numbers. Try to respond as quickly as you can while making as few mistakes as possible." 